\title{
Thermodynamics and weak cosmic censorship conjecture of the torus-like black hole
}

\author{
Yi-Wen Han, Xiao-Xiong Zeng ${ }^{\mathrm{a}}$, Yun Hong \\ Department of Physics, College of Computer Science and Electronic Information Engineering, Chongqing Technology and Business University, \\ Chongqing 400067, China
}

Received: 11 February 2019 / Accepted: 8 March 2019 / Published online: 20 March 2019

(C) The Author(s) 2019

\begin{abstract}
After studying the energy-momentum relation of charged particles' Hamilton-Jacobi equations, we discuss the laws of thermodynamics and the weak cosmic censorship conjecture in torus-like black holes. We find that both the first law of thermodynamic as well as the weak cosmic censorship conjecture are valid in both the normal phase space and extended phase space. However, the second law of thermodynamics is only valid in the normal phase space. Our results show that the first law and weak cosmic censorship conjecture do not depend on the phase spaces while the second law depends. What's more, we find that the shift of the metric function that determines the event horizon take the same form in different phase spaces, indicating that the weak cosmic censorship conjecture is independent of the phase space.
\end{abstract}

\section{Introduction}

Over the past few decades, great progress have been made in studying the thermodynamics of black holes. Theoretical physicists have studied the thermal and non-thermal radiation [1-5], entropy [6,7], thermodynamic phase transition [8-10], and thermodynamic properties of black holes in the frameworks of holography and geometry [11-18]. These results show that black hole thermodynamics is an interesting and important topic in the theory of gravity. And we know that black holes can be regarded as thermodynamic systems with Hawking temperature [19]. For the black hole thermodynamic systems, there is an irreducible mass distributed on the event horizon, which is similar to the thermodynamic entropy [20-24]. This similarity leads to the definition of the Bekenstein-Hawking entropy, which is found to be proportional to the area of the event horizon [25]. In addition, other conserved quantities such as the thermodynamic potential of a black hole is defined at its event horizon. In other words, the event horizon is essential for the thermodynamic system of the black hole.

In normal phase space of the thermodynamic systems, the cosmological constant that determines the spacetime asymptotic topology of the black hole is treated as a fixed parameter. However, according to the idea of Henneaux et al. [26], the cosmological constant can be treated as a dynamic variable in the thermodynamic system so that one can construct an extended phase space [27-31]. In the extended phase space, the cosmological constant $\Lambda$ was regarded as pressure $P$ [32] and its conjugate was regarded as volume with the definition $V=\left.(\partial M / \partial P)\right|_{S, J, Q}$. In this case, the first law of thermodynamics for black holes was revised to $d M=T d S+\Omega d J+\Phi d Q+V d P$. Here, $M$ is interpreted as enthalpy [33-35]. However, the validity of the first law of thermodynamics in the extended phase space does not mean the second law and the weak cosmic censorship conjecture are valid. Thus it is interesting and important to explore the validity of the second law and the weak cosmic censorship conjecture in the extended phase space of the black hole thermodynamic systems.

Recently, in the framework of extended phase space, [36] investigated laws of thermodynamics and weak cosmic censorship conjecture of a charged black hole. He found that the first law of thermodynamics was valid, and the second law was violated for extreme and near-extreme black holes. In addition, the weak cosmic censorship conjecture was found to be valid for the extremal black holes and the near-extremal black hole for they are stable. This work was then extended to the Born-Infeld gravity [37] and BTZ black holes [38], in which the contributions of other extensive quantities are considered besides the contributions of pressure and volume. They also claimed that they did not employ any approximations to deal with the weak cosmic censorship conjecture.

\footnotetext{
a e-mail: xxzengphysics@163.com
} 
In this paper, we intend to investigate laws of thermodynamics and the weak cosmic censorship conjecture of a toruslike black hole in the extended phase space. Different from the previous investigations, the topology of this spacetime is $S \times S \times M^{2}$. We intend to explore whether the topology will affect the weak cosmic censorship conjecture and the second law for they have not been touched until now. We will employ the idea in [36]. As a charged particle drops into the toruslike black hole, we first obtain the energy-momentum relation near the event horizon by solving the Hamilton-Jacobi equation. And based on this relation, laws of thermodynamics and weak cosmic censorship conjecture are investigated in both the normal phase space and extended phase space. We find both the first law and weak cosmic censorship conjecture are valid in the normal and extended phase space. However, the second law is violated in the extended phase space though it is valid in the normal phase space. Our result is consistent with that in [36], implying that the topology of the spacetime does not affect the thermodynamics and weak cosmic censorship conjecture.

The present work is organized as follows. In Sect. 2, the energy-momentum relation of the scalar particle is obtained by solving the Hamilton-Jacobi equation. In Sect. 3, thermodynamics and weak cosmic censorship conjecture are studied in the normal phase space. In Sect. 4, we investigated thermodynamics and weak cosmic censorship conjecture in the extended phase space. The conclusion is presented in Sect. 5.

\section{The dynamic behavior of charged particles in the thermodynamic system of the torus-like black hole}

The torus-like black hole solution is characterized by the line element [39]

$d s^{2}=-G(r) d t^{2}+G^{-1}(r) d r^{2}+r^{2}\left(d \theta^{2}+d \psi^{2}\right)$,

where

$G(r)=-\frac{\Lambda r^{2}}{3}-\frac{2 M}{\pi r}+\frac{4 Q^{2}}{\pi r^{2}}$.

For $\Lambda<0$, the metric has coordinate singularities at the horizon radii $r_{ \pm}$, which satisfy [40]

$$
-\frac{\Lambda r_{ \pm}^{2}}{3}-\frac{2 M}{\pi r_{ \pm}}+\frac{4 Q^{2}}{\pi r_{ \pm}^{2}}=0
$$

where, $r_{+}$and $r_{-}$correspond to outer and inner horizons of the black hole. The nonvanishing component of electromagnetic vector potential is

$A_{t}=-4 Q / r$

We first focus on the dynamics behavior of a charged particle swallowed by the black hole and its energy-momentum relationship near the event horizon. In the electric field, the motion of scattered particles satisfy the Hamilton-Jacobi equation of curved space-time, which is

$g^{\mu \nu}\left(p_{\mu}-e A_{\mu}\right)\left(p_{v}-e A_{v}\right)+\mu_{0}^{2}=0$,

where $\mu_{0}$ is rest mass of a scalar particle, $p_{\mu}$ the momentum of the particle, which is defined by

$p_{\mu}=\partial_{\mu} S$

here, $S$ is the Hamiltonian action. Considering the symmetry of the gravitational system, the action should be written as

$S=-E t+R(r)+\Theta(\theta)+L \psi$,

where, $E$ and $L$ are the energy and momentum of the particle, and they are conserved quantities of timespace in the gravitational system. From Eq. (1), the contravariant metric of the black hole can be written as

$$
\begin{aligned}
g^{\mu \nu} \partial_{\mu} \partial_{\nu}= & -G^{-1}(r)\left(\partial_{t}\right)^{2}+G(r)(\partial r)^{2}+r^{-2}\left(\partial_{\theta}\right)^{2} \\
& +(r \sin \theta)^{-2}(\partial \psi)^{2} .
\end{aligned}
$$

According to Eq. (7), the Hamilton-Jacobi equation (8) can be reduced to

$$
\begin{aligned}
& -G^{-1}(r)\left(E+e A_{t}\right)^{2}+G(r)\left(\partial_{r} R(r)\right)^{2}+r^{-2}\left(\partial_{\theta} \Theta(\theta)\right)^{2} \\
& +(r \sin \theta)^{-2} L^{2}+\mu_{0}^{2}=0 .
\end{aligned}
$$

Introducing a variable $\lambda$, we are able to make variable separation of the Eq. (9), the radial and angular components are

$$
\begin{aligned}
& -r^{2} G^{-1}(r)\left(-E-e A_{t}\right)^{2} \\
& \quad+r^{2} G(r)\left(\partial_{r} R(r)\right)^{2}+r^{2} \mu_{0}^{2}=-\lambda, \\
& \left(\partial_{\theta} \Theta(\theta)\right)^{2}+\sin ^{-2} \theta L^{2}=\lambda .
\end{aligned}
$$

With Eqs. (10) and (11), the radial and the angular components of the momentum can be expressed respectively as

$p^{r}=G(r) \sqrt{\frac{-\mu_{0}^{2} r^{2}+\lambda}{r^{2} G(r)}+\frac{1}{G(r)^{2}}\left(-E-e A_{t}\right)^{2}}$,

$p^{\theta}=\frac{1}{r^{2}} \sqrt{\lambda-\frac{1}{\sin ^{2} \theta} L^{2}}$.

Near the event horizon, the energy-momentum relation of charged particles is

$E=\frac{q}{r_{h}} e+\left|p^{r}\right|$

The charge has nothing to do with the direction of time, it only has relation with the interaction between the particle and the black hole. So, in order to ensure that the symbols of $E$ and $\left|p^{r}\right|$ are oriented towards the forward direction of time flow [20], we will select the positive sign in front of $\left|p^{r}\right|$. 


\section{Thermodynamics of the torus-like black hole in the normal phase space}

Now, we would like to investigate the thermodynamics of the torus-like black hole in normal phase space. According to the definition of surface gravity, the Hawking temperature of the black hole can be written as

$T=\frac{-12 Q^{2}+3 M r_{h}-\pi r_{h}^{4} \Lambda}{6 \pi^{2} r_{h}^{3}}$.

Using the relation between the Bekenstein-Hawking entropy and area, the entropy of the black hole can be expressed as

$S=\pi^{2} r_{h}^{2}$.

With Eq. (2), the relation between the mass and the event horizon of the torus-like black hole can be obtained as follows

$M=\frac{12 Q^{2}-\pi r_{h}^{4} \Lambda}{6 r_{h}}$.

When charged particles are scattered by the black hole, the energy and charge of the system should follow conservation laws. So, the internal energy and charge change of the black hole system should satisfy

$E=d M, \quad e=d Q$,

Substituting Eq. (4) and Eq. (18) into Eq. (14), we get

$d M=\Phi d Q+p^{r}$.

Similarly, the scattered particles will affect the event horizon of the black hole, leading to the change of the metric function $G(r)$. With the change of function $G(r), d M$ and $d Q$ can be deleted simultaneously. So, We can get $d r_{h}$ directly, and from Eq. (16), the variations of the black hole entropy can be expressed as

$d S_{h}=\frac{6 \pi^{2} p^{r} r_{h}^{3}}{-12 Q^{2}+3 M r_{h}-\pi \Lambda r_{h}^{4}}$.

By using Eqs. (15) and (20), we get $T d S_{h}=p^{r}$. Therefore the system internal energy in Eq. (19) can be rewritten as

$d M=T d S_{h}+\Phi d Q$

which is the first law of the torus-like black hole thermodynamics in the normal phase space.

By using Eq. (20), we also can discuss the second law of thermodynamics. In normal phase space, from the Eq. (15), we find $-12 Q^{2}+3 M r_{h}-\pi \Lambda r_{h}^{4}>0$, and put it into Eq. (20) we know $d S_{h}>0$. In the case, the change of the entropy of the black hole increases. In other words, the second law of thermodynamics for the torus-like black hole is valid in the normal phase space.

Of course, we can also test the weak cosmic censorship conjecture in normal phase space, which states that there should be a horizon to avoid the singularity to be naked.
Therefore, an event horizon is needed to guarantee the validity of the weak cosmic conjecture. To do this, we want to test whether an event horizon exists when a charged particle is absorbed by the black hole. In other words, whether there is a solution for function $G(r)$.

For the torus-like black hole, there is a minimum value for the function $G(r)$ with the radial coordinate $r_{\min }$. For the case $G\left(r_{\min }\right)>0$, there is not a horizon while for the case $G\left(r_{\min }\right) \leq 0$, there are horizons always. At $r_{\min }$, the following relations should be satisfied

$\left.G\right|_{r=r_{\text {min }}} \equiv G_{\text {min }}=\beta \leq 0$,

$\left.\partial_{r} G\right|_{r=r_{\text {min }}} \equiv G_{\text {min }}^{\prime}=0$,

$\left.\left(\partial_{r}\right)^{2} G\right|_{r=r_{\text {min }}} \equiv G_{m}^{\prime \prime}>0$.

For the extreme black hole, $\beta=0$. As a charged particle is sucked by the black hole, the changes in the conserved quantities such as the mass and charge of the black hole can be written as $(M+d M, Q+d Q)$. The locations of the minimum value and the event horizon can be written as $r_{\text {min }}+d r_{\text {min }}$, $r_{h}+d r_{h}$ respectively. Here, we have a transformation of the function $G(r)$, which is labeled as $d G_{\min }$. Using the condition $G_{\text {min }}^{\prime}=0$, at $r_{\text {min }}+d r_{\text {min }}, G(r)$ can be expressed as

$$
\begin{aligned}
\left.G\right|_{r=r_{\text {min }}+d r_{\text {min }}} & =G_{\text {min }}+d G_{\text {min }} \\
& =\beta+\left(\frac{\partial G_{\text {min }}}{\partial M} d M+\frac{\partial G_{\text {min }}}{\partial Q} d Q\right),
\end{aligned}
$$

For the extremal black hole, the horizon is located at $r_{\min }$, Eq. (19) therefore is valid at $r_{\min }$. Substituting Eq. (19) into Eq. (23), we find that $d Q$ is deleted at the same time. Equation (23) thus can be simplified to

$G_{\min }+d G_{\min }=-\frac{2 p^{r}}{\pi r_{\min }}$.

Equation (24) shows that $G\left(r_{\min }+d r_{\text {min }}\right)$ is smaller than $G\left(r_{\min }\right)$ when a charged particle is swallowed by the black hole. This result will cause extremal black holes to be transformed into non-extremal black holes. That is, the weak cosmic censorship conjecture is valid in the normal phase space.

\section{Thermodynamics and weak cosmic censorship conjecture in extended phase space}

Next, we turn to investigate the thermodynamics and weak cosmic censorship conjecture in extended phase space. Namely the cosmological constant $\Lambda$ will be treated as the thermodynamic pressure, and its conjugate as the thermodynamic volume, which can be expressed as respectively

$$
P=-\Lambda /(8 \pi), \quad V=4 \pi^{2} r_{h}^{3} / 3 .
$$


From Eqs. (15), (16), (25), and $\Phi=4 Q / r_{h}$, we obtain the Smarr formula

$M=2(T S-V P)+\Phi Q$.

Considering that $M$ has the physical significance of enthalpy [32] in extended phase space, it has relation to the system internal energy as

$M=U+P V$.

According to the laws of conversion, when a particle is swallowed by the black hole, the change in the conserved quantity of the system should be equal to the energy and charge of the particle. Therefore, in extended phase space, Eq. (18) can be rewritten as

$E=d U=d(M-P V), \quad e=d Q$.

Correspondingly, the energy in Eq. (19) changes into

$d U=\Phi d Q+\left|p^{r}\right|$.

Similarly, the absorbed particle must also change the event horizon of the black hole due to the backreaction. But the event horizon is always determined by function $G(r)$. In the new horizon, $r_{h}+d r_{h}$, there is also a relation $G\left(r_{h}+d r_{h}\right)=$ $G\left(r_{h}\right)+d G_{h}=0$, which means

$$
\begin{aligned}
d G_{h}= & \frac{\partial G_{h}}{\partial M} d M+\frac{\partial G_{h}}{\partial Q} d Q \\
& +\frac{\partial G_{h}}{\partial \Lambda} d \Lambda+\frac{\partial G_{h}}{\partial r_{h}} d r_{h}=0 .
\end{aligned}
$$

With Eq. (28), the Eq. (29) can be rewritten as

$$
d M-d(P V)=\Phi d Q+\left|p^{r}\right| \text {. }
$$

From Eq. (31), we can obtain

$d \Lambda=\frac{24 Q d Q-6 r_{h} d M+6 p^{r} r_{h}-3 \pi \Lambda r_{h}^{3} d r_{h}}{\pi r^{4}}$.

Substituting Eq. (32) into Eq. (30), we can delete $d Q$ and $d M$ directly. So, there is only a relation between $\left|p^{r}\right|$ and $d r_{h}$, which yields

$d r_{h}=\frac{6 p^{r} r_{h}^{2}}{-24 Q^{2}+6 M r_{h}+\pi \Lambda r_{h}^{4}}$.

Based on this relation, the variations of entropy and volume of the black hole can be expressed as

$$
\begin{aligned}
d S_{h} & =\frac{12 \pi^{2} p^{r} r_{h}^{3}}{-24 Q^{2}+6 M r_{h}+\pi \Lambda r_{h}^{4}}, \\
d V & =\frac{24 \pi^{2} p^{r} r_{h}^{4}}{-24 Q^{2}+6 M r_{h}+\pi \Lambda r_{h}^{4}} .
\end{aligned}
$$

Using the above formulas, we find $T d S_{h}-P d V=\left|p^{r}\right|$. The internal energy in Eq. (29) thus would change into

$d U=\Phi d Q+T d S_{h}-P d V$.
With Eq. (27), we can obtain the relation between the enthalpy and internal energy in extended phase space, that is

$d M=d U+P d V+V d P$.

Substituting Eq. (37) into Eq. (36), we get

$d M=T d S_{h}+\Phi d Q+V d P$,

which is the first law in extended phase space. That is, in the extended phase space, the first law of thermodynamics still holds as a charged particle is absorbed by the black hole.

We also can discuss the second law of thermodynamics in extended phase space. We would consider the case of extreme black holes firstly. By using the condition $T=0$, we can obtain $r_{h}$ from Eq. (15) and substitute $r_{h}$ into Eq. (34), the change of the black hole entropy is given by

$d S_{\text {extremal }}=\frac{4 \pi p^{r}}{\Lambda r_{h}}$.

In Eq. (39), we know $d S_{h}<0$ for $\Lambda<0$. This result shows that change of the entropy of the torus-like black hole is always decreased. That is, the second law of thermodynamics is violated for the extremal black hole.

For the non-extremal black holes, we can get $Q$ with Eq. (2), that is

$Q=\frac{\sqrt{r_{h}} \sqrt{6 M+\pi r_{h}^{3} \Lambda}}{2 \sqrt{3}}$.

Substituting this equation into Eq. (34), we find

$d S_{h}=-\frac{12 \pi^{2} p^{r} r_{h}^{2}}{6 M+\pi r_{h}^{3} \Lambda}$.

It is obvious that $d S_{h}$ is negative for we know $6 M+\pi r_{h}^{3} \Lambda$ is positive from Eq. (40). The second law of thermodynamics is also violated for the non-extremal black hole.

Similarly, we also can examine the weak cosmic censorship conjecture in extended phase space. Considering the backreaction, and using Eq. (22), the conserved quantity such as mass $M$, charge $Q$, and cosmological constant $\Lambda$ will change into $(M+d M, Q+d Q, \Lambda+d \Lambda)$ as a charged particle drops into the black hole. The locations of the minimum value and the event horizon will then change into $r_{\min }+d r_{\min }$, $r_{h}+d r_{h}$. At $r_{\text {min }}+d r_{\text {min }}$, the change of function $G(r)$ should satisfy

$\left.G\right|_{r=r_{\text {min }}+d r_{\min }}=\left(\frac{\partial G_{\min }}{\partial M} d M+\frac{\partial G_{\min }}{\partial Q} d Q+\frac{\partial G_{\min }}{\partial \Lambda} d \Lambda\right)$.

For the extremal black hole, the event horizon is located at $r_{\text {min }}$, Eq. (29) thus is applicable at $r_{\text {min }}$. Substituting Eq. (29) 
into Eq. (42), we get

$G_{\text {min }}+d G_{\text {min }}=-\frac{2 p^{r}}{\pi r_{\text {min }}}+\Lambda r_{\text {min }} d r_{\text {min }}$.

By using $G_{\min }^{\prime}=0$, we can get the mass of the black hole, and further

$$
\begin{aligned}
d M= & \frac{4 d Q Q}{r_{\min }}-\frac{1}{6} d \Lambda \pi r_{\min }^{3}+d r_{\min } \\
& \times\left(-\frac{2}{3} \pi r_{\min }^{2} \Lambda-\frac{12 Q^{2}-\pi r_{\min }^{4} \Lambda}{6 r_{\min }^{2}}\right) .
\end{aligned}
$$

In addition, according to $f\left(r_{h}=r_{\min }\right)=0$, we can obtain

$d r_{\min }=-\frac{r_{\min }\left(24 d Q Q+d \Lambda \pi r_{\min }^{4}\right)}{3\left(-4 Q^{2}+\pi r_{\min }^{4} \Lambda\right)}$.

At the new lowest point, there is also a relation

$\left.\partial_{r} G\right|_{r=r_{\text {min }}+d r_{\text {min }}}=G_{\text {min }}^{\prime}+d G_{\text {min }}^{\prime}=0$.

which means

$d G_{\text {min }}^{\prime}=\frac{\partial G_{\text {min }}^{\prime}}{\partial Q} d Q+\frac{\partial G_{\text {min }}^{\prime}}{\partial \Lambda} d \Lambda+\frac{\partial G_{\text {min }}^{\prime}}{\partial r_{\text {min }}} d r_{\text {min }}=0$

From Eqs. (31), (44), (45), (47), we know that there are four equations with respect to $d M, d Q, d r_{\text {min }}, d \Lambda$. We can get $d r_{\min }$ by solving them. Interestingly, we find $d r_{\min }=0$. Thus Eq. (43) can be simplified as

$G_{\min }+d G_{\min }=-\frac{2 p^{r}}{\pi r_{\min }}$

which is the same as that in Eq. (24). That is, as the contribution of the $P V$ term is considered, the weak cosmic censorship conjecture is also valid. Interestingly, the shift of the metric function that determines the event horizon take the same form in both the normal phase space and extended phase space.

\section{Conclusion}

In our work, by solving the Hamilton-Jacobi equation, we first obtained the energy-momentum relation near the horizon as a charged particle drops into the torus-like black hole. Then we got the first law of thermodynamics in both the normal and extended phase space. It should be noted that though some work $[34,35]$ have studied the thermodynamics of a class of black holes in extended phase space. However, their work is premised on the existence of the first law of thermodynamics. While in this paper, we investigated the dynamics of the scalar particles under the scattering of black holes. Our work proves the rationality of the previous work [34,35].

The validity of the first law of thermodynamics doesn't mean the second law is valid. Therefore, we investigated the change of the entropy of the extremal and non-extremal black holes in the normal and extended phase space. We found that the change of entropy for both the extremal and non-extremal black holes were positive in the normal phase space while negative in the extended phase space, which indicates that the second law is valid in the normal phase space and violated in the extended phase space. The reason causing the difference stem from the contribution of pressure and volume, though the physical mechanism is not clear.

Finally, we investigated the weak cosmic oversight hypothesis by examining the metric function that determines the horizon of the black hole at the minimum point in both the normal and extended phase space. As a charged particle is swallowed, we found that the changes of the metric function were negative in both the normal and extended phase space. In this case, there are always solutions for the toruslike black hole so that the singularity of the spacetime is hidden, the weak cosmic censorship conjecture thus is valid always. Interestingly, we found that the shift of the metric function take the same form in both the normal phase space and extended phase space, which indicates that the weak cosmic censorship conjecture is independent of the phase space. The extremal black holes in both phase space thus would change into non-extremal black holes. Our result is different from that in [36], where the author found that the extremal black holes would be extremal black holes in the extended phase space. The reason maybe be from that we does not employ any approximation while [36] employed.

Acknowledgements This work is supported by the National Natural Science Foundation of China (Grant nos. 11875095), and Basic Research Project of Science and Technology Committee of Chongqing (Grant no. cstc2018jcyjA2480).

Data Availability Statement This manuscript has no associated data or the data will not be deposited [Authors' comment: All the results are obtained analytically.]

Open Access This article is distributed under the terms of the Creative Commons Attribution 4.0 International License (http://creativecomm ons.org/licenses/by/4.0/), which permits unrestricted use, distribution, and reproduction in any medium, provided you give appropriate credit to the original author(s) and the source, provide a link to the Creative Commons license, and indicate if changes were made. Funded by SCOAP ${ }^{3}$.

\section{References}

1. S.W. Hawking, Phys. Rev. D 13, 191 (1976)

2. J.Y. Zhang, Z. Zhao, J. High Energy Phys. 10, 055 (2005)

3. J.Y. Zhang, J.H. Fan, Phys. Lett. 648, 133 (2007)

4. X.X. Zeng, S.Z. Yang, Gen. Relativ. Gravit. 40, 2107 (2008)

5. X.X. Zeng, J.S. Hou, S.Z. Yang, Pramana 70, 409 (2008)

6. G. 't Hooft, Nucl. Phys. B 256, 727 (1985)

7. J.L. Cardy, Nucl. Phys. B 270, 186 (1986)

8. M.R. Setare, Phys. Rev. D 70, 087501 (2004)

9. M. Cavaglia, S. Das, Class. Quantum Gravity 21, 4511 (2004) 
10. P. Chen, R.J. Adler, Nucl. Phys. Proc. Suppl. 124, 103 (2003)

11. X.X. Zeng, X.M. Liu, B.W. Liu, J. High Energy Phys. 03, 031 (2014)

12. X.X. Zeng, H. Zhang, L.F. Li, Phys. Lett. B 756, 170 (2016)

13. X.X. Zeng, D.Y. Chen, L.F. Li, Phys. Rev. D 91, 046005 (2015)

14. R.G. Cai, X.X. Zeng, H.Q. Zhang, JHEP 1707, 082 (2017)

15. Y.W. Han, J.Y. Zhang, Phys. Lett. B 692, 74 (2010)

16. Y.W. Han, G. Chen, Phys. Lett. B 714, 127 (2012)

17. G. Ruppeiner, Phys. Rev. D 75, 024037 (2007)

18. G. Ruppeiner, Phys. Rev. D 78, 024016 (2008)

19. S.W. Hawking, Commun. Math. Phys. 43, 199 (1975)

20. D. Christodoulou, Phys. Rev. Lett. 25, 1596 (1970)

21. J.M. Bardeen, Nature 226, 64 (1970)

22. B. Gwak, Phys. Rev. D 95(12), 124050 (2017)

23. B. Gwak, JHEP 1809, 081 (2018)

24. B. Gwak, Entropy 20(11), 855 (2018)

25. J.D. Bekenstein, Phys. Rev. D 7, 2333 (1973)

26. M. Henneaux, C. Teitelboim, Commun. Math. Phys. 98, 391 (1985)

27. M.M. Caldarelli, G. Cognola, D. Klemm, Class. Quantum Gravity 17, 399235(2000)
28. S.H. Hendi, A. Sheykhi, S. Panahiyan, B.E. Panah, Phys. Rev. D 92, 064028 (2015)

29. Y. Sekiwa, Phys. Rev. D 73, 084009 (2006)

30. R. Banerjee, S.K. Modak, D. Roychowdhury, J. High Energy Phys. 1210, $125240(2012)$

31. S. Wang, S.Q. Wu, F. Xie, L. Dan, Chin. Phys. Lett. 23, 1096 (2006)

32. S.R. Dastor, J. Traschen, Class. Quantum Gravity 26, 195011 (2009)

33. B.P. Dolan, Class. Quantum Gravity 28, 235017 (2011)

34. B.P. Dolan, Class. Quantum Gravity 28, 125020 (2011)

35. D. Kubiznak, R.B. Mann, J. High Energy Phys. 1207, 033245 (2012)

36. B. Gwak, J. High Energy Phys. 1711, 129 (2017)

37. X.X. Zeng, H.Q. Zhang (2019). arXiv:1901.04247 [hep-th]

38. X.X.Zeng, Y.W. Han, D.Y. Chen (2019). arXiv: 1901.08915 [gr-qc]

39. C.G. Huang, C.B. Liang, Phys. Lett. A 201, 27 (1995)

40. M. Sharif, W. Javed, Can. J. Phys. 90, 903 (2012) 